\title{
Role of hepatectomy in gastric cancer with multiple liver-limited metastases
}

\author{
Hiromichi Shirasu ${ }^{1}$ Takahiro Tsushima ${ }^{1} \cdot$ Masahiro Kawahira $^{1} \cdot$ Sadayuki Kawai $^{1}$ • \\ Takeshi Kawakami $^{1}$ - Yosuke Kito ${ }^{1}$. Yukio Yoshida ${ }^{1}$ - Satoshi Hamauchi ${ }^{1}$. \\ Akiko Todaka $^{1} \cdot$ Tomoya Yokota $^{1} \cdot$ Nozomu Machida $^{1} \cdot$ Kentaro Yamazaki $^{1}$. \\ Akira Fukutomi $^{1} \cdot$ Yusuke Onozawa $^{3}$ - Masanori Terashima ${ }^{2} \cdot$ Katsuhiko Uesaka $^{4}$. \\ Hirofumi Yasui ${ }^{1}$
}

Received: 15 February 2017/Accepted: 25 May 2017/Published online: 2 June 2017

(c) The International Gastric Cancer Association and The Japanese Gastric Cancer Association 2017

\begin{abstract}
Background Several studies have demonstrated the benefit of hepatectomy for treating gastric cancer (GC) with liverlimited metastases (LLM). The survival benefit of hepatectomy compared with that of systemic chemotherapy is unknown, particularly in patients with multiple LLM. This study investigated the survival benefit of hepatectomy compared with that of systemic chemotherapy administered to patients with GC with multiple LLM.

Methods We retrospectively reviewed the data of consecutive patients with GC with two or three LLM who underwent hepatectomy or received systemic chemotherapy as initial treatment at the Shizuoka Cancer Center between December 2004 and December 2015.

Results Nine of 24 patients who met the inclusion criteria underwent hepatectomy, and 15 received chemotherapy. In the hepatectomy group, all patients achieved $\mathrm{R} 0$ resection and none died during hospitalization. Three patients received adjuvant chemotherapy. Disease recurred in eight patients (88.9\%). In the chemotherapy group, three patients underwent hepatectomy following initial chemotherapy and did not experience recurrence or death during follow-
\end{abstract}

Takahiro Tsushima

t.tsushima@scchr.jp

1 Division of Gastrointestinal Oncology, Shizuoka Cancer Center, 1007 Shimonagakubo Nagaizumi-cho Sunto-gun, Shizuoka 411-8777, Japan

2 Division of Gastric Surgery, Shizuoka Cancer Center, Suntogun, Shizuoka, Japan

3 Division of Medical Oncology, Shizuoka Cancer Center, Sunto-gun, Shizuoka, Japan

4 Division of Hepato-Biliary-Pancreatic Surgery, Shizuoka Cancer Center, Sunto-gun, Shizuoka, Japan up. Median follow-up was 47.9 months and median overall survival (OS) was 38.1 and 24.8 months in the chemotherapy and hepatectomy groups, respectively. Multivariate analysis of OS, including initial treatment, revealed that unilobar liver metastasis was the only independent favorable prognostic factor.

Conclusions Although hepatectomy for patients with GC with multiple LLM is not recommended as the initial therapy, it prolonged the survival of patients with tumors controlled using systemic chemotherapy.

Keywords Gastric cancer - Liver metastases · Hepatectomy · Chemotherapy

\section{Introduction}

Gastric cancer (GC) is the third leading cause of cancerrelated deaths worldwide [1]. The survival outcome in locally advanced GC is markedly improved through treatment using radical surgery, D2 lymph node dissection, and perioperative systemic chemotherapy [2-8]. In contrast, GC with distant metastasis represented by peritoneal seeding, liver metastasis, and para-aortic lymph node involvement has poor survival outcomes, with a median survival time of 10-14 months [9-11].

Although systemic chemotherapy is the standard treatment for recurrent or metastatic advanced GC [12], several studies suggest a benefit of hepatectomy for GC with liverlimited metastases (LLM), i.e., when there are no other distant metastases [13-23]. According to these results, hepatectomy should be empirically considered for patients with a few LLM. However, those studies found that worse survival outcomes are associated with patients with multiple LLM compared with those with solitary LLM. The 
efficacy of hepatectomy in GC with multiple LLM is unknown. At our institution, hepatectomy was performed only for patients with solitary liver metastases before 2011, consistent with previous studies reporting favorable survival outcomes. Accordingly, since 2011, we have extended the application of hepatectomy to cases with up to three liver metastases. We conducted this study to investigate the survival benefit of hepatectomy compared with that of systemic chemotherapy administered to patients with GC with two or three LLM.

\section{Methods}

We retrospectively reviewed the data of consecutive GC patients with LLM who underwent hepatectomy or received systemic chemotherapy as the initial treatment at the Shizuoka Cancer Center between December 2004 and December 2015. The inclusion criteria were as follows: histologically confirmed adenocarcinoma, ECOG performance status (PS) 0-1, two or three liver metastases (synchronous or metachronous), no distant metastasis other than the liver, technically resectable disease (primary GC and regional lymph nodes were considered resectable in patients with synchronous liver metastasis), adequate organ function (white blood cell count, $\geq 3.0 \times 10^{9}$ and $<12.0 \times 10^{9}$, platelet count, $\geq 100 \times 10^{9} / \mathrm{L}$; aspartate aminotransferase, $<100 \mathrm{U} / \mathrm{L}$; alanine aminotransferase, $<100 \mathrm{U} / \mathrm{L}$; serum bilirubin, $<2.0 \mathrm{mg} / \mathrm{dL}$; serum creatinine, $<1.2 \mathrm{mg} / \mathrm{dL}$ ), no history of chemotherapy or radiotherapy, and no other malignancies within the past 5 years. We excluded patients who were refractory to adjuvant chemotherapy, which was defined as recurrence within 6 months from the last administration of adjuvant chemotherapy. Histopathological descriptions were in accordance with the 14th edition of the Japanese Classification of Gastric Carcinoma. The decision to perform hepatectomy was made according to the consensus opinion of a dedicated multidisciplinary team that assessed tumor and patient characteristics such as extent of the disease, organ function, and comorbidity. Contrast-enhanced computed tomography (CT) and contrast-enhanced magnetic resonance imaging (MRI) were performed in all cases as preoperative assessments. The Shizuoka Cancer Center Institutional Review Board approved this study, and informed consent was obtained from the patients.

\section{Statistical analysis}

OS was defined as the time from diagnosis until death from any cause. Progression-free survival (PFS) was defined as the time from diagnosis to tumor relapse, progression, or death. Comparisons between categorical variables were performed using Fisher's exact test. The median values of the variables were compared using the Mann-Whitney $U$ test. Survival curves were generated using the KaplanMeier method. The log-rank test was used to evaluate differences among survival curves. Multivariate analysis using the Cox proportional hazards model (backward stepwise selection) was employed to evaluate the associations between clinical variables and survival. A $p$ value of $<0.05$ was considered statistically significant. Statistical analysis was conducted using EZR version 1.32 (Saitama Medical Center, Jichi Medical University, Saitama, Japan) [24].

\section{Results}

\section{Patient selection and characteristics}

Of the 284 GC patients with LLM, 226 were excluded because they had more than three liver metastases. Other reasons for exclusion were solitary metastasis $(n=27)$, history of therapy $(n=5)$, poor PS $(n=1)$, and concomitant malignancy $(n=1)$. The data for 24 patients were analyzed. Nine patients underwent hepatectomy and 15 received chemotherapy as the initial treatment. The clinicopathological characteristics of the patients are presented in Table 1. Patients who received chemotherapy were younger than those who underwent hepatectomy, and all the other characteristics were almost the same in both groups.

\section{Treatment outcomes of the hepatectomy group $(n=9)$}

All patients who underwent hepatectomy achieved R0 resection as follows: partial hepatectomy, $n=8$ and segmentectomy, $n=1$. Gastrectomy with D2 lymph node resection was performed for all patients with synchronous LLM. Postoperative complications are listed in Table 2. No deaths occurred during hospitalization. One patient died from an unknown cause one month after hepatectomy. Three patients received S-1 adjuvant chemotherapy after hepatectomy. The other patients $(n=6)$ did not receive adjuvant chemotherapy for the following reasons: deterioration of the patient's performance status after hepatectomy $(n=3)$, refusal $(n=2)$, and intolerance to previous adjuvant therapy $(n=1)$.

Eight patients $(88.9 \%)$ experienced recurrent disease. Recurrence patterns were multiple LLM $(n=4)$, solitary LLM $(n=2)$, liver with lymph node metastases $(n=1)$, and liver with peritoneal metastases $(n=1)$. One patient with a solitary liver-limited recurrence underwent a second hepatectomy and survived without recurrence. Three 
Table 1 Patients' characteristics

\begin{tabular}{|c|c|c|c|}
\hline Characteristics & Hepatectomy $(n=9)$ & Chemotherapy $(n=15)$ & $p$ value \\
\hline Gender & & & 1 \\
\hline Male & 8 & 14 & \\
\hline Female & 1 & 1 & \\
\hline Age, median (range) & $74(64-81)$ & $59(42-76)$ & 0.0021 \\
\hline Performance status (ECOG) & & & 0.042 \\
\hline 0 & 6 & 15 & \\
\hline 1 & 3 & 0 & \\
\hline Histology & & & 0.118 \\
\hline Differentiated & 9 & 10 & \\
\hline Undifferentiated & 0 & 5 & \\
\hline \multicolumn{4}{|l|}{ HER2 status } \\
\hline Positive & 0 & 2 & \\
\hline Negative & 0 & 5 & \\
\hline Unknown & 9 & 8 & \\
\hline \multicolumn{4}{|l|}{ Liver metastases } \\
\hline Number of metastases & & & 0.052 \\
\hline 2 & 9 & 9 & \\
\hline 3 & 0 & 6 & \\
\hline Tumor location & & & 0.403 \\
\hline Unilobar & 5 & 5 & \\
\hline Bilobar & 4 & 10 & \\
\hline Timing of metastases & & & 1 \\
\hline Synchronous & 6 & 10 & \\
\hline Metachronous & 3 & 5 & \\
\hline Median sum of diameters of metastases (mm) & $25(10-70)$ & $31(10-130)$ & 0.742 \\
\hline Median CEA level (ng/mL) & $4.1(1.7-579.7)$ & $4.8(0.8-424.9)$ & 1 \\
\hline $\mathrm{RMH}$ & & & 0.356 \\
\hline Good & 5 & 12 & \\
\hline Moderate & 4 & 3 & \\
\hline Poor & 0 & 0 & \\
\hline
\end{tabular}

ECOG Eastern Cooperative Oncology Group, HER2 human epidermal growth factor receptor type 2, CEA carcinoembryonic antigen, $R M H$ Royal Marsden Hospital prognostic score

patients received chemotherapy as follows: irinotecan plus cisplatin $(n=2)$ and S-1 monotherapy $(n=1)$. Four patients only received palliative supportive care because of poor PS and older age.

\section{Treatment outcomes of the chemotherapy group $(n=15)$}

Eleven patients in the chemotherapy group received fluoropyrimidine plus cisplatin. Trastuzumab was included in the regimen of two patients with HER2-positive tumors. Three patients received S-1 monotherapy, and one patient received irinotecan plus cisplatin. Three patients underwent conversion hepatectomy (hepatectomy following initial chemotherapy).
The treatment course in each of three patients was as follows. Patient 1, a 42-year-old man, was diagnosed as having HER2-positive advanced GC with three liver metastases. After three cycles of S-1 and cisplatin combined with trastuzumab chemotherapy, partial response was achieved. Total gastrectomy with segmentectomy was then performed. Patient 2, a 53 year-old man, was diagnosed as having two metachronous liver metastases after curative gastrectomy. Four cycles of S-1 and cisplatin therapy were administered. With significant regression of the known lesions and no evidence of a new metastatic lesion, segmentectomy was performed. Patient 3, a 51 year-old man, was diagnosed as having HER2-positive advanced GC with three liver metastases. Capecitabine and cisplatin combined with trastuzumab chemotherapy were administered. The primary and metastatic 
Table 2 Operative details and postoperative outcomes of hepatectomy

\begin{tabular}{ll}
\hline Variables & \\
\hline Type of hepatectomy & 8 \\
Partial hepatectomy & 1 \\
Segmentectomy & 9 \\
Radical surgery (R0 resection) & 16 (9-34) \\
Median (range) postoperative hospital stay (days) $^{\text {a }}$ & \\
Postoperative complications $^{\text {a }}$ & 5 \\
Absent & 4 \\
Present & 3 \\
Intraabdominal abscess & 1 \\
Anastomotic leakage & 1 \\
Bile leakage & 1 \\
Wound infection & 1 \\
Peripheral catheter infection & 0 \\
Postoperative mortality &
\end{tabular}

${ }^{\text {a }}$ Grade II or higher according to the Clavien-Dindo classification

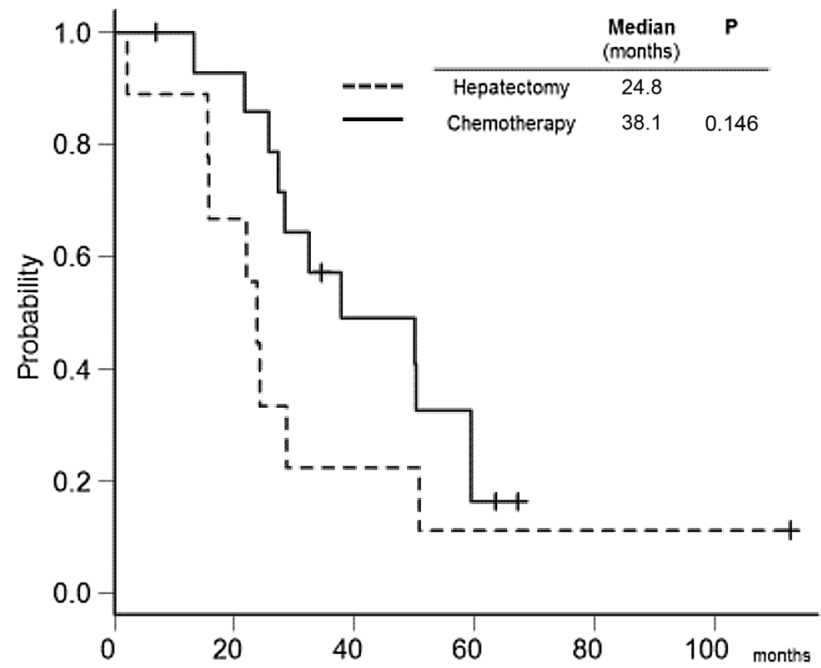

Fig. 1 Overall survival curves of patients who received initial hepatectomy (dashed line) and chemotherapy (solid line)

lesions exhibited a partial response after eight cycles; total gastrectomy with partial hepatectomy was then performed. Two of the three patients received adjuvant chemotherapy.

Twelve patients received second-line chemotherapy as follows: paclitaxel $(n=5)$, docetaxel $(n=1)$, irinotecan plus cisplatin $(n=4)$, irinotecan monotherapy $(n=1)$, and fluoropyrimidine plus methotrexate $(n=1)$.

\section{Survival and prognostic factors}

During a follow-up period of 47.9 months, the median length of OS was 38.1 and 24.8 months for the chemotherapy and surgery groups, respectively $(p=0.146$ ) (Fig. 1$)$, and

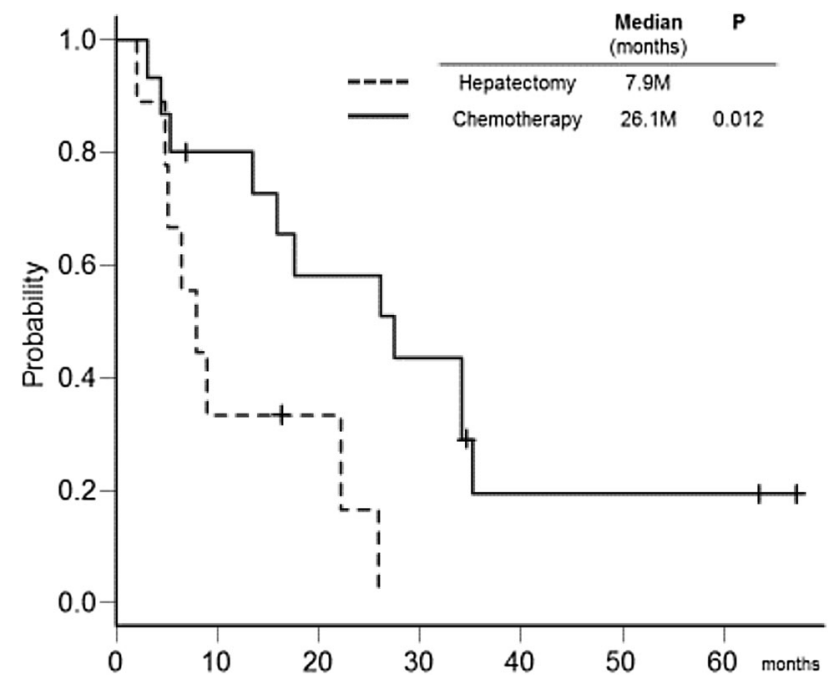

Fig. 2 Progression-free survival curves of patients who received initial hepatectomy (dashed line) and chemotherapy (solid line)

2-year OS values were 85.7 and $44.4 \%$ in the chemotherapy and surgery groups, respectively. The median PFS of the chemotherapy group was 26.1 months, and that of the surgery group was 7.9 months $(p=0.012)$ (Fig. 2). Notably, none of the three patients who underwent hepatectomy following initial chemotherapy experienced recurrence or death during follow-up (Fig. 3).

The only variable potentially associated with longer survival, as revealed by univariate analysis, was unilobar liver metastases, which was identified as the only independent favorable prognostic factor by multivariate analysis ( $p=0.044$; hazard ratio, $0.37 ; 95 \%$ confidence interval: 0.14-0.98) (Table 3).

\section{Discussion}

In the current study, we showed that initial hepatectomy for patients with GC with two or three LLM does not yield a survival benefit compared with initial chemotherapy. However, radical hepatectomy following chemotherapy may be associated with a favorable outcome. Further, unilobar LLM predicted a favorable outcome in this group.

Surgery with curative intent is generally not indicated for liver metastases of GC; however, several reports demonstrate a survival benefit of hepatectomy for GC with liver metastases. The median OS ranges from 11 months to 49 months, and the 5-year survival rate is $13-37 \%$ [13-23]. Although each report reflects the experience of a single institution with a small number of cases, an accumulation of such positive data supports hepatectomy as the preferred treatment option for selected patients. In the Japanese gastric cancer treatment guidelines, a multidisciplinary approach, including hepatectomy, is described as an 


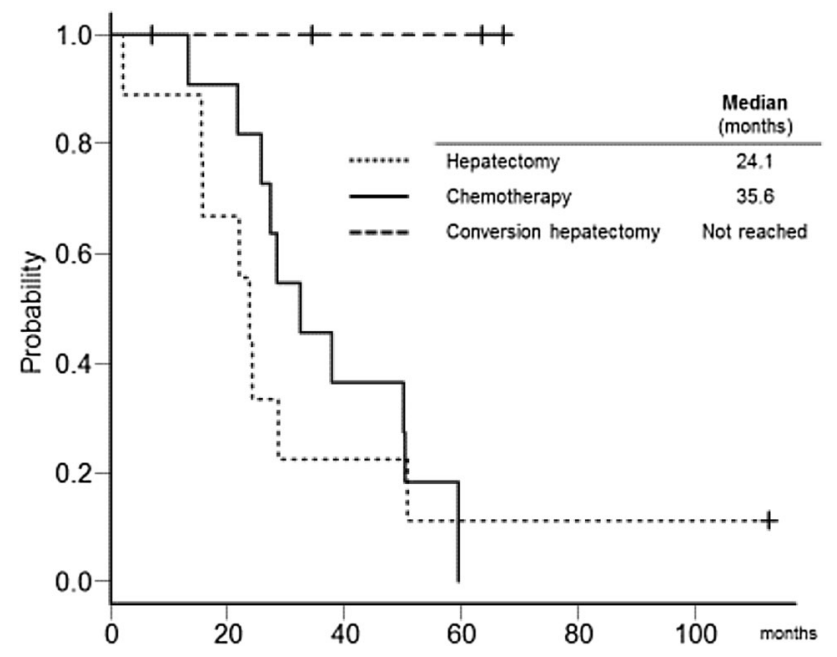

Fig. 3 Overall survival curves of patients who underwent conversion hepatectomy, i.e., radical hepatectomy after initial chemotherapy (dashed line), who received only chemotherapy (solid line), and who underwent initial hepatectomy (dotted line)

optional approach for patients with LLM when the number of metastatic nodules is small and other noncurative factors are absent [12].

The indication of hepatectomy for GC with liver metastases is not firmly established because of a paucity of evidence. Previous clinical studies demonstrate that the expansion of the resection area does not improve the survival of patients with GC [25-27]. Patient selection is crucial, considering the invasiveness of hepatectomy, and the goal should be long-term survival. In many retrospective reports on hepatectomy for liver metastases from GC, solitary LLM is the most common favorable prognostic factor [13-23]. In contrast, three or more liver metastases and a liver tumor diameter $\geq 5 \mathrm{~cm}$ are independent predictors of poor survival [15]. These results suggest that GC with solitary LLM is a good candidate for hepatectomy, but its indication as a treatment option for multiple LLM remains unclear.

Liver metastases of GC are generally aggressive [28]. A French multicenter study found that hepatectomy for GC achieves a 5-year survival rate of 15-30\% [29]. In our study, 8/9 patients who underwent hepatectomy as initial therapy had intrahepatic recurrence and their 5-year OS was $11.1 \%$. These results indicate the presence of a high incidence of occult micrometastases at the time of hepatectomy. Systemic chemotherapy is a reasonable choice for the initial treatment for GC with multiple LLM. First, preoperative chemotherapy is theoretically useful for identifying nonresponders to chemotherapy or the presence of a highly aggressive tumor and helps to avoid futile attempts to perform surgery. Second, preoperative chemotherapy may be effective for eradicating these micrometastases. And third, the tolerability of preoperative chemotherapy is generally better than that of postoperative chemotherapy [8]. In our study, patients who underwent initial hepatectomy may be at risk of physical status deterioration, which leads to low acceptability of adjuvant therapy: three patients were unable to receive adjuvant chemotherapy because of a deterioration in their performance status after hepatectomy. The three patients in the chemotherapy group who underwent hepatectomy subsequent to chemotherapy did not experience recurrence during the observation period. In addition, liver metastases in these patients were unilobar. Some studies suggest that the existence of unilobar liver metastases is a favorable prognostic factor for hepatectomy administered to patients with GC, which was observed in our study as well [30, 31].

Table 3 Univariate and multivariate analytical results for overall survival

\begin{tabular}{|c|c|c|c|c|c|c|}
\hline \multirow[t]{2}{*}{ Variables } & \multicolumn{3}{|c|}{ Univariate analysis } & \multicolumn{2}{|c|}{ Multivariate analysis } & \multirow[t]{2}{*}{$p$} \\
\hline & Hazard ratio & $95 \% \mathrm{CI}$ & $p$ & Hazard ratio & $95 \% \mathrm{CI}$ & \\
\hline Age $<70$ vs. $\geq 70$ & 0.57 & $0.23-1.44$ & 0.238 & & & \\
\hline Performance status 0 vs. 1 & 0.70 & $0.28-1.77$ & 0.450 & & & \\
\hline Histology differentiated vs. undifferentiated & 2.15 & $0.62-7.47$ & 0.227 & & & \\
\hline \multicolumn{7}{|l|}{ Liver metastases } \\
\hline Number of metastases 2 vs. 3 & 0.88 & $0.28-2.70$ & 0.817 & & & \\
\hline Synchronous vs. metachronous & 2.00 & $0.72-5.51$ & 0.183 & & & \\
\hline Unilobar vs. bilobar & 0.34 & $0.13-0.91$ & 0.030 & 0.37 & $0.14-0.98$ & 0.044 \\
\hline Sum of diameter $<30$ vs. $\geq 30 \mathrm{~mm}$ & 1.02 & $0.41-2.53$ & 0.962 & & & \\
\hline CEA level $<10$ vs. $\geq 10 \mathrm{ng} / \mathrm{mL}$ & 0.83 & $0.30-2.30$ & 0.715 & & & \\
\hline RMH good vs. moderate & 0.36 & $0.13-1.00$ & 0.051 & & & \\
\hline Hepatectomy vs chemotherapy & 2.09 & $0.83-5.27$ & 0.153 & & & \\
\hline
\end{tabular}

$C E A$ carcinoembryonic antigen, $R M H$ Royal Marsden Hospital prognostic score 
The present study has several limitations. First, this study was a small sample-sized, retrospective study at a single institution. Further, there must have been some inherent selection bias in our analyses. In fact, there were significant differences in PS and age between the two groups. This may have affected outcomes, particularly the poor survival outcomes in the hepatectomy group. Second, there is no established surgical strategy for technically resectable LLM from GC because of a paucity of data. We discussed every case, and the final decision was made by a multidisciplinary conference held every week.

Despite these limitations, we believe that the results of this study are worthy of discussion, because this is the first report to investigate the $\mathrm{OS}$ of patients with GC with multiple LLM who underwent hepatectomy or chemotherapy.

The results of the present study suggest that hepatectomy could be a component of the multidisciplinary strategy for this population, although the optimal duration of preoperative chemotherapy is unclear. Previous studies for locally advanced GC showed the efficacy of 8-12 weeks of preoperative chemotherapy, whereas a longer duration of preoperative treatment was not associated with longer overall survival [8, 32-35]. Based on these results, preoperative chemotherapy within 12 weeks can be recommended. Several trials of surgical intervention for GC with single and multiple LLM are now ongoing (UMIN000011445, NCT02578368, NCT02380131). Subgroup analyses of these prospective studies will help reveal the significance of hepatectomy with or without chemotherapy for multiple LLM compared with single LLM. In conclusion, hepatectomy for patients with GC with two or three LLM may lead to long-term survival in patients whose tumors respond to systemic chemotherapy, although it is not recommended as initial therapy.

Acknowledgements The authors thank the patients and their families for their participation in this study.

\section{Compliance with ethical standards}

Conflict of interest The authors declare that they have no conflict of interest.

Ethical standards All procedures followed were in accordance with the ethical standards of the responsible committee on human experimentation (institutional and national) and with the Helsinki Declaration of 1964 and later versions. Informed consent or a substitute for it was obtained from all patients before they were included in the study.

\section{References}

1. Ferlay JSI, Ervik M, Dikshit R, Eser S, Mathers C, Rebelo M. GLOBOCAN 2012 v1.0, Cancer Incidence and Mortality
Worldwide: IARC CancerBase No. 11. Lyon: International Agency for Research on Cancer; 2013. Available from: http:// globocan.iarc.fr, Accessed on 9/2/2017.

2. de Aretxabala X, Konishi K, Yonemura Y, Ueno K, Yagi M, Noguchi M, et al. Node dissection in gastric cancer. Br J Surg. 1987;74(9):770-3.

3. Maruyama K, Okabayashi K, Kinoshita T. Progress in gastric cancer surgery in Japan and its limits of radicality. World J Surg. 1987;11(4):418-25.

4. Sasako M, McCulloch P, Kinoshita T, Maruyama K. New method to evaluate the therapeutic value of lymph node dissection for gastric cancer. Br J Surg. 1995;82(3):346-51.

5. Songun I, Putter H, Kranenbarg EM, Sasako M, van de Velde CJ. Surgical treatment of gastric cancer: 15-year follow-up results of the randomised nationwide Dutch D1D2 trial. Lancet Oncol. 2010;11(5):439-49.

6. Sakuramoto S, Sasako M, Yamaguchi T, Kinoshita T, Fujii M, Nashimoto A, et al. Adjuvant chemotherapy for gastric cancer with S-1, an oral fluoropyrimidine. $\mathrm{N}$ Engl $\mathrm{J}$ Med. 2007;357(18):1810-20.

7. Noh SH, Park SR, Yang H-K, Chung HC, Chung I-J, Kim S-W, et al. Adjuvant capecitabine plus oxaliplatin for gastric cancer after D2 gastrectomy (CLASSIC): 5-year follow-up of an openlabel, randomised phase 3 trial. Lancet Oncol. 2014;15(12):1389-96.

8. Cunningham D, Allum WH, Stenning SP, Thompson JN, Van de Velde CJ, Nicolson M, et al. Perioperative chemotherapy versus surgery alone for resectable gastroesophageal cancer. $\mathrm{N}$ Engl $\mathrm{J}$ Med. 2006;355(1):11-20.

9. Yamada Y, Higuchi K, Nishikawa K, Gotoh M, Fuse N, Sugimoto N, et al. Phase III study comparing oxaliplatin plus S-1 with cisplatin plus S-1 in chemotherapy-naive patients with advanced gastric cancer. Ann Oncol. 2015;26(1):141-8.

10. Koizumi W, Narahara H, Hara T, Takagane A, Akiya T, Takagi M, et al. S-1 plus cisplatin versus S-1 alone for first-line treatment of advanced gastric cancer (SPIRITS trial): a phase III trial. Lancet Oncol. 2008;9(3):215-21.

11. Bang YJ, Van Cutsem E, Feyereislova A, Chung HC, Shen L, Sawaki A, et al. Trastuzumab in combination with chemotherapy versus chemotherapy alone for treatment of HER2-positive advanced gastric or gastro-oesophageal junction cancer (ToGA): a phase 3, open-label, randomised controlled trial. Lancet. 2010;376(9742):687-97.

12. Japanese Gastric Cancer Association. Japanese gastric cancer treatment guidelines 2014 (ver. 4). Gastric Cancer. 2016;20(1):1-19. doi:10.1007/s10120-016-0622-4.

13. Tatsubayashi T, Tanizawa Y, Miki Y, Tokunaga M, Bando E, Kawamura T, et al. Treatment outcomes of hepatectomy for liver metastases of gastric cancer diagnosed using contrast-enhanced magnetic resonance imaging. Gastric Cancer. 2016;20(2). doi:10. 1007/s10120-016-0611-7 (Epub 2016/05/09).

14. Tiberio GA, Ministrini S, Gardini A, Marrelli D, Marchet A, Cipollari C, et al. Factors influencing survival after hepatectomy for metastases from gastric cancer. Eur J Surg Oncol. 2016;42(8):1229-35. doi:10.1016/j.ejso.2016.03.030 (Epub 2016/05/03).

15. Kinoshita T, Kinoshita T, Saiura A, Esaki M, Sakamoto H, Yamanaka T. Multicentre analysis of long-term outcome after surgical resection for gastric cancer liver metastases. Br J Surg. 2015;102(1):102-7.

16. Oki E, Tokunaga S, Emi Y, Kusumoto T, Yamamoto M, Fukuzawa K, et al. Surgical treatment of liver metastasis of gastric cancer: a retrospective multicenter cohort study (KSCC1302). Gastric Cancer. 2016;19(3):968-76.

17. Takemura N, Saiura A, Koga R, Arita J, Yoshioka R, Ono Y, et al. Long-term outcomes after surgical resection for gastric 
cancer liver metastasis: an analysis of 64 macroscopically complete resections. Langenbecks Arch Surg. 2012;397(6):951-7.

18. Wang YN, Shen KT, Ling JQ, Gao XD, Hou YY, Wang XF, et al. Prognostic analysis of combined curative resection of the stomach and liver lesions in 30 gastric cancer patients with synchronous liver metastases. BMC Surg. 2012;12:20.

19. Schildberg CW, Croner R, Merkel S, Schellerer V, Muller V, Yedibela $\mathrm{S}$, et al. Outcome of operative therapy of hepatic metastatic stomach carcinoma: a retrospective analysis. World $\mathrm{J}$ Surg. 2012;36(4):872-8.

20. Garancini M, Uggeri F, Degrate L, Nespoli L, Gianotti L, Nespoli A, et al. Surgical treatment of liver metastases of gastric cancer: is local treatment in a systemic disease worthwhile? HPB (Oxford). 2012;14(3):209-15.

21. Tsujimoto H, Ichikura T, Ono S, Sugasawa H, Hiraki S, Sakamoto N, et al. Outcomes for patients following hepatic resection of metastatic tumors from gastric cancer. Hepatol Int. 2010;4(1):406-13.

22. Koga R, Yamamoto J, Ohyama S, Saiura A, Seki M, Seto Y, et al. Liver resection for metastatic gastric cancer: experience with 42 patients including eight long-term survivors. Jpn J Clin Oncol. 2007;37(11):836-42.

23. Okano K, Maeba T, Ishimura K, Karasawa Y, Goda F, Wakabayashi $\mathrm{H}$, et al. Hepatic resection for metastatic tumors from gastric cancer. Ann Surg. 2002;235(1):86-91.

24. Kanda Y. Investigation of the freely available easy-to-use software 'EZR' for medical statistics. Bone Marrow Transplant. 2013;48(3):452-8.

25. Sano T, Sasako M, Mizusawa J, Yamamoto S, Katai H, Yoshikawa $\mathrm{T}$, et al. Randomized controlled trial to evaluate splenectomy in total gastrectomy for proximal gastric carcinoma. Ann Surg. 2017;265(2):277-283. doi:10.1097/sla.0000000000001814.

26. Sasako M, Sano T, Yamamoto S, Kurokawa Y, Nashimoto A, Kurita A, et al. D2 lymphadenectomy alone or with para-aortic nodal dissection for gastric cancer. $N$ Engl $J$ Med. 2008;359(5):453-62.

27. Kurokawa Y, Sasako M, Sano T, Yoshikawa T, Iwasaki Y, Nashimoto A, et al. Ten-year follow-up results of a randomized clinical trial comparing left thoracoabdominal and abdominal transhiatal approaches to total gastrectomy for adenocarcinoma of the oesophagogastric junction or gastric cardia. Br J Surg. 2015;102(4):341-8.

28. Kodera Y, Fujitani K, Fukushima N, Ito S, Muro K, Ohashi N, et al. Surgical resection of hepatic metastasis from gastric cancer: a review and new recommendation in the Japanese gastric cancer treatment guidelines. Gastric Cancer. 2014;17(2):206-12.

29. Adam R, Chiche L, Aloia T, Elias D, Salmon R, Rivoire M, et al. Hepatic resection for noncolorectal nonendocrine liver metastases: analysis of 1,452 patients and development of a prognostic model. Ann Surg. 2006;244(4):524-35.

30. Sakamoto Y, Sano T, Shimada K, Esaki M, Saka M, Fukagawa T, et al. Favorable indications for hepatectomy in patients with liver metastasis from gastric cancer. J Surg Oncol. 2007;95(7):534-9.

31. Makino H, Kunisaki C, Izumisawa Y, Tokuhisa M, Oshima T, Nagano $\mathrm{Y}$, et al. Indication for hepatic resection in the treatment of liver metastasis from gastric cancer. Anticancer Res. 2010;30(6):2367-76.

32. Tsuburaya A, Mizusawa J, Tanaka Y, Fukushima N, Nashimoto A, Sasako M. Neoadjuvant chemotherapy with S-1 and cisplatin followed by D2 gastrectomy with para-aortic lymph node dissection for gastric cancer with extensive lymph node metastasis. Br J Surg. 2014;101(6):653-60.

33. Honma Y, Yamada Y, Terazawa T, Takashima A, Iwasa S, Kato $\mathrm{K}$, et al. Feasibility of neoadjuvant S-1 and oxaliplatin followed by surgery for resectable advanced gastric adenocarcinoma. Surg Today. 2016;46(9):1076-82.

34. Park I, Ryu MH, Choi YH, Kang HJ, Yook JH, Park YS, et al. A phase II study of neoadjuvant docetaxel, oxaliplatin, and S-1 (DOS) chemotherapy followed by surgery and adjuvant S-1 chemotherapy in potentially resectable gastric or gastroesophageal junction adenocarcinoma. Cancer Chemother Pharmacol. 2013;72(4):815-23.

35. Yoshikawa T, Morita S, Tanabe K, Nishikawa K, Ito Y, Matsui T, et al. Survival results of a randomised two-by-two factorial phase II trial comparing neoadjuvant chemotherapy with two and four courses of S-1 plus cisplatin (SC) and paclitaxel plus cisplatin (PC) followed by D2 gastrectomy for resectable advanced gastric cancer. Eur J Cancer. 2016;62:103-11. 